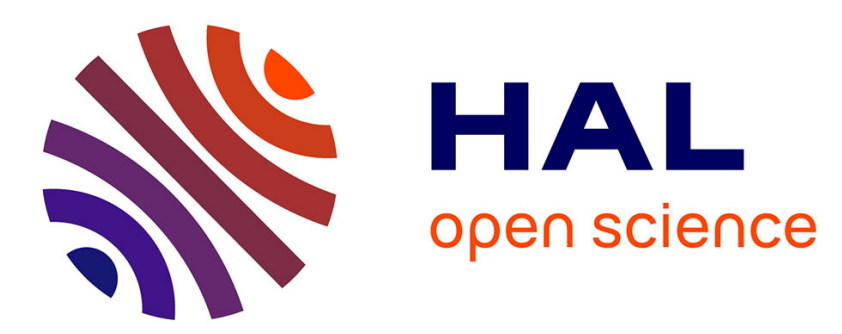

\title{
Nanoforce sensing with magnetic springs using differential approach to compensate external mechanical disturbances.
}

Margot Billot, Emmanuel Piat, Joël Abadie, Joel Agnus, Philippe Stempfle

\section{- To cite this version:}

Margot Billot, Emmanuel Piat, Joël Abadie, Joel Agnus, Philippe Stempfle. Nanoforce sensing with magnetic springs using differential approach to compensate external mechanical disturbances.. International Conference on Robotics and Automation, May 2016, Stockolm, Sweden. hal-02868198

\section{HAL Id: hal-02868198 \\ https://hal.science/hal-02868198}

Submitted on 15 Jun 2020

HAL is a multi-disciplinary open access archive for the deposit and dissemination of scientific research documents, whether they are published or not. The documents may come from teaching and research institutions in France or abroad, or from public or private research centers.
L'archive ouverte pluridisciplinaire $\mathbf{H A L}$, est destinée au dépôt et à la diffusion de documents scientifiques de niveau recherche, publiés ou non, émanant des établissements d'enseignement et de recherche français ou étrangers, des laboratoires publics ou privés. 


\title{
Nanoforce sensing with magnetic springs using a differential approach to compensate external mechanical disturbances
}

\author{
Margot Billot ${ }^{1}$, Emmanuel Piat ${ }^{1}$, Joël Abadie ${ }^{1}$, Joël Agnus ${ }^{1}$, Philippe Stempflé ${ }^{1}$
}

\begin{abstract}
Nanoforce sensors using passive magnetic springs associated to a macroscopic seismic mass are known to be a possible alternative to force sensors based on elastic microstructures like Atomic Force Microscopes if the nanoforces that have to be measured are characterized by a bandwidth limited to a few Hertz. The estimation of the unknown force applied to the seismic mass is based on the deconvolution of the noisy measurement of the mass displacement which has an under-damped dynamic. Despite their high performances in terms of linearity, resolution and measurement range, such force sensors are extremely sensitive to low frequency environmental mechanical disturbances. They are also sensitive to the temperature evolution of the ambient air. The evaluation, modeling and compensation of such environmental disturbances have to be specifically studied in the context of magnetic springs associated to a macroscopic seismic mass. This article presents an estimation and a passive compensation strategy of the low frequency and non-stationary mechanical disturbances that is based on a differential principle. This approach is applied to a nanoforce sensor based on diamagnetic levitation developed in the last decade and gives an uncertainty below the nanonewton level.
\end{abstract}

\section{ADDRESSED PROBLEM}

\section{A. Nanoforce sensors and environmental disturbances}

Achieving progresses in the measurement of micro and nanoforce remains a necessity to enhance the performances of micro and nanorobotics devices and to characterize, in terms of adhesion and friction, functionalized and/or structured surfaces of material involved in microrobotics applications such as micro-assembling or dexterous micromanipulation. Such progresses concern both force sensors embedded in microrobotics devices and force sensors embedded in dedicated measurement platforms. Whatever is their final use, all micro or nanoforce sensors use a transducer to convert the force into a measurable effect. In many force sensors, this effect is related to the displacement $x$ of a force-displacement transducer. Most classical designs are based on elastic microstructures with one or several degrees of freedom which have a high resonant frequency: AFM based microforce sensors [1] [2], piezoresistive microforce sensors [3] [4], capacitive microforce sensors [5], piezoelectric microforce sensors [6]. All these micro and nano force sensors are submitted to external disturbances that affect their dynamic and should be estimated and compensated if their bandwidth and amplitude corrupt the force that has to be estimated. A possible categorization of such environmental disturbances and their domains of influence can be found in

\footnotetext{
${ }^{1}$ Femto-ST Institute, Besançon - France

CNRS - UFC - ENSMM - UTBM

epiat@ens $2 \mathrm{~m}$. fr
}

[7]. Their estimation and active or passive compensation in micro/nanoforce sensors remains an open research problem.

\section{B. Nanoforce sensors using magnetic springs}

Nanoforce sensors with low resonant frequency that are based on low stiffness magnetic springs associated to a macroscopic seismic mass are a possible alternative to classical designs based on elastic microstructures when the force bandwidth that has to be measured is limited to a few Hertz. The unknown force is applied on a macroscopic seismic mass connected to a passive magnetic spring and induces a displacement response of the mass. Because of the macroscopic size of the force-displacement transducer, these force sensors, developed in the last decade, are commonly used in force measurement macroscopic platforms with one [8] or several degrees of freedom [9]. They have been used for instance with success to characterize the mechanical behavior of human ovocytes whose stiffness is commonly below $0.01 \mathrm{~N} / \mathrm{m}$ [10]. The unknown force estimation has been addressed in the past for a nanoforce sensor based on diamagnetic levitation [8] [11] [12] but without taking into account the environmental disturbances acting on the transducer, like the angular variations of the anti-vibration table supporting the device or the residual seismic vibrations that are not filtered by the table. The seismic mass, called maglevtube, is a ten centimeter-long capillary tube stuck on two small magnets $M_{2}$ (see Fig. 1). It is levitating passively around a given equilibrium state thanks to the diamagnetic levitation principle. The measurement $x_{k}^{m}$ of the position $x$ of the maglevtube is done with a confocal chromatic sensor. It is aimed at the deflector located at the rear of the maglevtube. The complete estimation process of the force $\vec{F}^{x}$ acting on the maglevtube (Fig. 1) is fully developed in [12]. This deconvolution approach based on a Kalman filtering only takes into account the dynamic of the maglevtube and not the environmental disturbances. Two main low frequency external mechanical disturbances have been identified and are modeled in the next section III. Section III presents the passive differential approach that is used on the force sensor based on diamagnetic levitation in order to compensate these disturbances. Section IV presents simulated results and the final section deals with experimental results introducing thermal influence.

\section{Maglevtube Modeling With LOW-FREQUenCy MECHANICAL DISTURBANCES}

Let $G$ be the center of gravity of the maglevtube and $x$ its position in the frame $R_{t}$ (shown in Fig. 2) attached to 


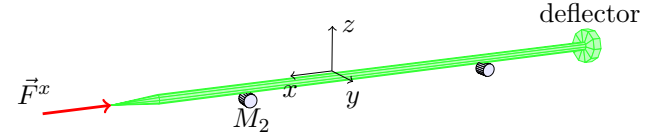

Fig. 1: Macroscopic seismic mass used as force-displacement transducer (maglevtube).

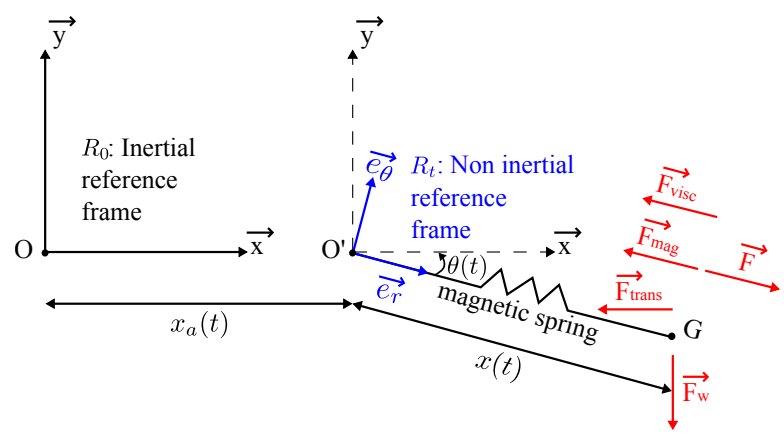

Fig. 2: Reference frames, coordinates and forces applied to the maglevtube.

the tabletop of the anti-vibration table (base of the sensor). Coordinate $x$ is therefore the longitudinal displacement of the maglevtube (along $\overrightarrow{e_{r}}$ direction in Fig. 2) measured with the confocal chromatic sensor located on the tabletop. The value $x$ is set to zero when the maglevtube is in steady state without any external excitation. The frame $R_{t}$ is a non-inertial reference frame because of the low frequency vibrations transmitted by the ground to the tabletop. These vibrations are only considered in the direction of the measurement. As shown in Fig. 2, let note $\theta(t)$ the rotation angle (corresponding to the angular variation of the antivibration table) between $R_{0}$ (attached to the laboratory) and $R_{t}$. The anti-vibration table oscillates with a very low frequency. Therefore the angular acceleration of $R_{t}$ respect to $R_{0}$ is closed to zero and is supposed to be negligible $(\ddot{\theta}(t)=0$, $\dot{\theta}(t)=0$ and $\theta(t)=\theta)$. Let note $x_{a}(t)$ the position of the table expressed in $R_{0}$ which is an unmeasured quantity contrary to $x(t)$. The fundamental principle of dynamic applied in the non-inertial reference frame $R_{t}\left(O^{\prime}, \overrightarrow{e_{r}}, \overrightarrow{e_{\theta}}, \overrightarrow{e_{z}}\right)$ is given by:

$$
m \vec{a}_{G_{/ R_{t}}}=\sum \vec{F}+\vec{F}_{\text {trans }}+\vec{F}_{\text {centr }}+\vec{F}_{\text {Cor }}+\vec{F}_{\text {Eul }}
$$

in which $m$ is the maglevtube mass, $\vec{a}_{G_{/ R_{t}}}$ is the transducer mass acceleration along $\overrightarrow{e r}, \sum \vec{F}$ is the sum of all the external forces applied to the maglevtube and $\vec{F}_{\text {trans }}, \vec{F}_{\text {cent }}$, $\vec{F}_{C o r}$ and $\vec{F}_{E u l}$ are the translation, centrifugal, Coriolis and Euler force, respectively. In our case, as $\ddot{\theta}$ and $\dot{\theta}$ are assumed to be zero, $\vec{F}_{\text {cent }}=\vec{F}_{C o r}=\vec{F}_{E u l}=\overrightarrow{0}$ and $\vec{a}_{G_{/ R t}}=\ddot{x} \cdot \overrightarrow{e r}$. Moreover, $\vec{F}_{\text {trans }}=-m \vec{a}_{0^{\prime} R_{t}}=\left[\begin{array}{c}-m \ddot{x}_{a} \cos \theta \\ -m \ddot{x}_{a} \sin \theta \\ 0\end{array}\right]_{/ R_{t}}$, with $\vec{a}_{0^{\prime} R_{t}}$ the acceleration of $R_{t}$ respect to $R_{0}$. Then, $\sum \vec{F}=$ $\vec{F}+\vec{F}_{w}+\vec{F}_{\text {mag }}+\vec{F}_{v i s c}$ with $\vec{F}, \vec{F}_{w}, \vec{F}_{m a g}$ and $\vec{F}_{v i s c}$ the unknown external force to be measured, the weight, the return magnetic force (magnetic spring) and the viscous

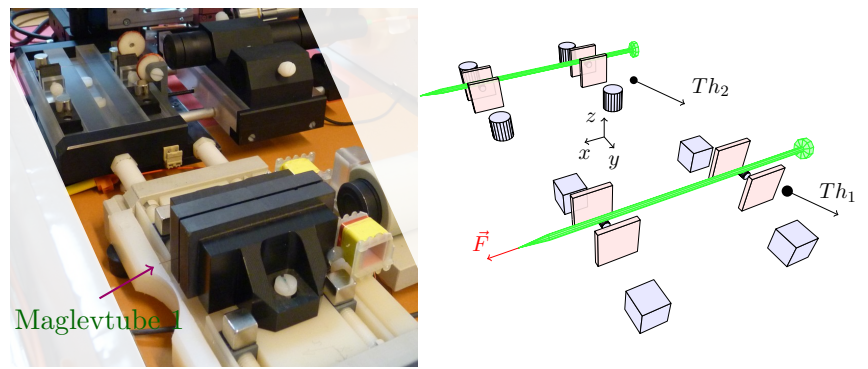

Fig. 3: Differential force sensor prototype using two maglevtubes (described en [8]).

friction force (mainly due to the air friction against the rear deflector), respectively. Assuming that the unknown force and the tube displacement are collinear to $\overrightarrow{e_{r}}$, that the latter displacement remains in the linear domain [8] and that its speed is small, the forces are defined by:

$$
\begin{aligned}
& \vec{F}=\left[\begin{array}{c}
F^{x} \\
0 \\
0
\end{array}\right]_{/ R_{t}} \quad \vec{F}_{w}=\left[\begin{array}{c}
m g \sin \theta \\
-m g \cos \theta \\
0
\end{array}\right]_{/ R_{t}} \\
& \vec{F}_{m a g}=-K_{m} x \overrightarrow{e_{r}} \quad \vec{F}_{v i s c}=-K_{v} \dot{x} \overrightarrow{e_{r}}
\end{aligned}
$$

where $g$ is the gravity acceleration, $K_{m}$ is the magnetic stiffness, $K_{v}$ the viscous damping coefficient. All the nonzero forces applied to the maglevtube are represented in Fig. 2. The fundamental principle of the dynamics (1), in projection along $\overrightarrow{e_{r}}$, becomes:

$$
m \ddot{x}+K_{v} \dot{x}+K_{m} x=F^{x}+m\left(g \sin \theta-\ddot{x}_{a} \cos \theta\right) .
$$

Equation (4) shows that the dynamic of the maglevtube in the reference frame $R_{t}$ depends on the force $F^{x}(t)$ and the disturbances $\theta$ and $\ddot{x}_{a}(t)$. For instance, with $m$ equal to 20 mg, a tiny angle $\theta$ equal to $5 \mu \mathrm{rad}$ leads to a disturbance force $m g \sin \theta$ equal to $1 \mathrm{nN}$.

\section{FORCE ESTIMATION BY DIFFERENTIAL DECONVOLUTION}

The deconvolution framework developed in [12] gives a way to estimate the unknown input of a second-order dynamical system knowing a noisy measurement of its output. By applying this approach, based on Kalman Filtering, to Eq. (4), it gives a way to compute an estimation of the unknown input

$$
\mathscr{F}^{x}=F^{x}+m\left(g \sin \theta-\ddot{x}_{a} \cos \theta\right)
$$

knowing a measurement of $x$. In order to deduce an estimation of $F^{x}$ with the estimation of $\mathscr{F}^{x}$, it is necessary to compensate the effects of the disturbances induced by $\theta$ and $\ddot{x}_{a}$ in the previous equation (5). A possible solution could be the direct measurement of these disturbances using adequate sensors like inclinometers and accelerometers with sufficient resolution at low frequency. Instead of this, a differential principle is developed in this article. This principle avoids to use of new types of sensors in the measurement chain by using two distinct maglevtubes and enables to compensate 


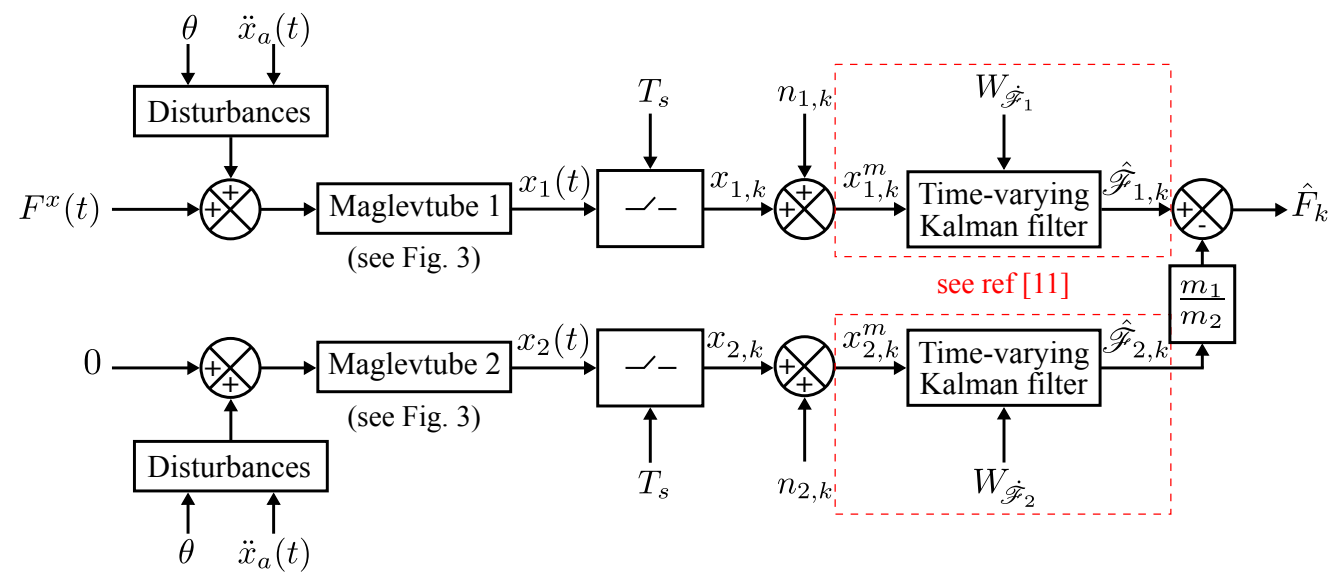

Fig. 4: Diagram of differential principle that enables the passive rejection of the disturbances.

all the common mechanical disturbance which are applied on these two maglevtubes (even if not modelized). The two force sensors are placed side-by-side on the same anti-vibration table (see Fig. 3) and enclosed in a chamber. Therefore, because the table rigidity is supposed to be infinite at low frequency, it is assumed that the two sensors are submitted at the same time to the same perturbations due to table movements $\left(\theta\right.$ and $\left.\ddot{x}_{a}(\mathrm{t})\right)$. The first maglevtube is dedicated to the measurement of the external force applied on its tip, whereas the second one is only excited by the perturbations. These perturbations can be estimated and used to compensate the ones applied on the first maglevtube. Using Eq. (4) from Section [I] the dynamic of the two maglevtubes are described by the following equations:

$$
\begin{gathered}
m_{1} \ddot{x}+K_{v_{1}} \dot{x}+K_{m_{1}} x=F^{x}+m_{1}\left(g \sin \theta-\ddot{x}_{a} \cos \theta\right) \\
m_{2} \ddot{x}+K_{v_{2}} \dot{x}+K_{m_{2}} x=m_{2}\left(g \sin \theta-\ddot{x}_{a} \cos \theta\right)
\end{gathered}
$$

where $m_{i}, K_{v_{i}}$ and $K_{m_{i}}$ are the mass, the magnetic stiffness and the viscous damping coefficient of the maglevtube $i$, respectively $(i=1,2)$. The diagram from Fig. 4 allows to estimate the force applied to the maglevtube 1 compensating the mechanical disturbances. Both signals $\eta_{1, k}$ and $\eta_{2, k}$ are gaussian measurement noises. The compensation is deduced from (6) and (7). Indeed, the time-varying Kalman filter corresponding to the maglevtube 1 gives $\mathscr{\mathscr { F }}_{1, k}$ which is an estimation at time $t_{k}=k T_{s}$ of the right-hand-side in (6), i.e. $F^{x}+m_{1}\left(g \sin \theta-\ddot{x}_{a} \cos \theta\right)$. The Kalman filter is parametrized by a scalar power spacial density $W_{\dot{F}_{1}}$ (see [12] for details). Moreover, the time-varying Kalman filter corresponding to the maglevtube 2 gives the estimation $\hat{\mathscr{F}}_{2, k}$ of $m_{2}(g \sin \theta-$ $\left.\ddot{x}_{a} \cos \theta\right)$. Therefore, it is just necessary to multiply $\hat{\mathscr{F}}_{2, k}$ by the known ratio $\frac{m_{1}}{m_{2}}$ (contrary to an elastic microstructure, each maglevtube mass is easily measurable) and to subtract the result from $\hat{\mathscr{F}}_{1, k}$ to obtain $\hat{F}_{k}$ the estimation of $F^{x}$ at time $k T_{s}$. This rejection approach of the perturbation due to $\theta$ and $\ddot{x}_{a}(t)$ using a mechanical differential design is quite similar in its principle to a common mode rejection usually used in electronics and, as previously said, does not necessitate the measurement of $\theta$ and $\ddot{x}_{a}(t)$ with dedicated sensors with high resolution at low frequency.

\section{Simulated RESUltS}

In order to illustrate the influence of the modeled disturbances $\theta$ and $\ddot{x}_{a}(t)$, the complete prototype presented in Section III is simulated using Matlab-Simulink with the following parameters:

- quasi-static sinusoidal variation of the tabletop angle $\theta$ with a frequency of $0.0005 \mathrm{~Hz}$;

- mono-modal variation of the table position $x_{a}(t)$ with a frequency of $0.8 \mathrm{~Hz}$;

- sampling rate $T_{s}$ of $0.001 \mathrm{~s}$;

- $R_{1}$ and $R_{2}$ the variance of the displacement sensors equal to $4.10^{-16} \mathrm{~m}^{2}$;

- Characteristics of the maglevtube 1: $m_{1}=21.40 \mathrm{mg}$, $K_{m_{1}}=0.0120 \mathrm{~N} / \mathrm{m}, K_{\nu_{1}}=8.5026 .10^{-6}$;

- Characteristics of the maglevtube $2: m_{2}=21.58 \mathrm{mg}$, $K_{m_{2}}=0.0149 \mathrm{~N} / \mathrm{m}, K_{\nu_{2}}=1.1848 .10^{-5}$;

- Kalman filters adjustments: $W_{\dot{\mathscr{F}}_{1}}=1 \cdot 10^{-18} \mathrm{~N}^{2} / \mathrm{Hz}$ and $W_{\dot{\mathscr{F}}_{2}}=1.10^{-18} \mathrm{~N}^{2} / \mathrm{Hz}$.

Figure 5 shows the result of this simulation for a zero force and a step force $F^{x}$ set to an amplitude of $10 \mathrm{nN}$. Without disturbances rejection, one can see the global and very low frequency drift due to the disturbance $m_{1} g \sin \theta$ on which is added the disturbance $m_{1} \ddot{x}_{a} \cos \theta$ at a higher frequency. These disturbances are correctly rejected using the differential deconvolution. Figure 5 also illustrates that this rejection is totally independant of the measured force $F^{x}(t)$ (here equal to 0 or $10 \mathrm{nN}$ ) because, as shown by Eq. (6), the mechanical disturbances considered are inputs added to $F^{x}(t)$ and the deconvolution process is linear.

\section{EXPERIMENTAL MEASUREMENTS}

\section{A. Influence of temperature}

Figure 6 shows experimental results obtained with the prototype presented in Section I-A It gives the force estimation $\hat{F}_{k}$ when there is no external force applied on the maglevtube 


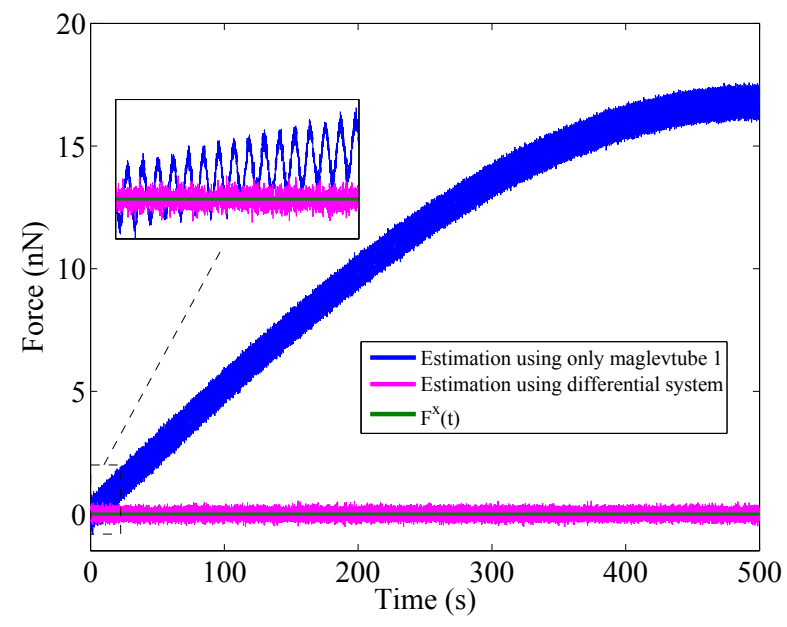

(a) $F^{x}(t)=0 \mathrm{nN}$

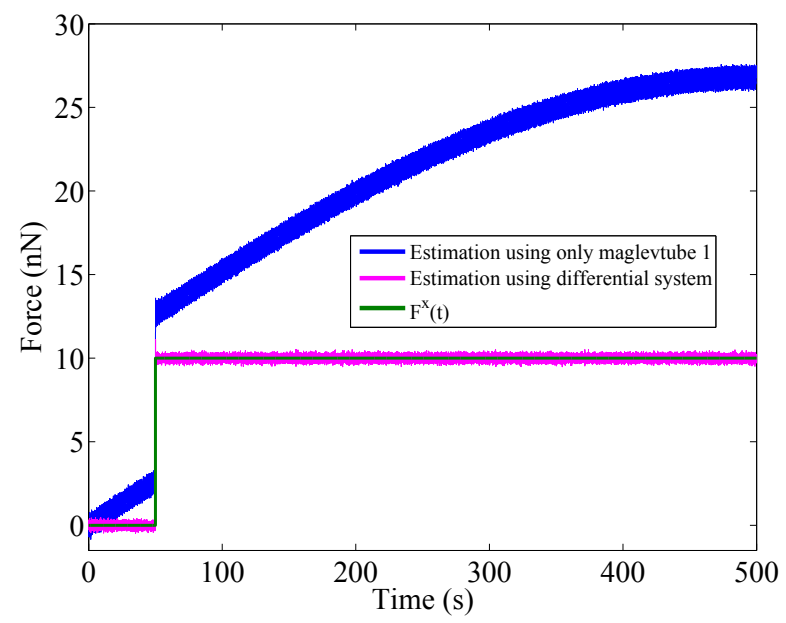

(b) $F^{x}(t)=10 \mathrm{nN}$

Fig. 5: Simulation of force estimation versus time for an external force $F^{x}(t)$ with environmental disturbances that are rejected or not.

tip, thus $F^{x}(t)$ is equal to 0 . In this case, the dynamic of both maglevtubes are given by:

$$
\begin{aligned}
& m_{1} \ddot{x}+K_{v_{1}} \dot{x}+K_{m_{1}} x=m_{1}\left(g \sin \theta-\ddot{x}_{a} \cos \theta\right) \\
& m_{2} \ddot{x}+K_{v_{2}} \dot{x}+K_{m_{2}} x=m_{2}\left(g \sin \theta-\ddot{x}_{a} \cos \theta\right)
\end{aligned}
$$

It can be seen that, contrary to what was expected with the simulation results (Fig. 5), the zero force estimated in the nanonewton scale using the differential principle does not stay close to zero. This is due to the fact that the two deconvolutions process (light blue and dark blue) do not give the same estimation of the input $m_{1}\left(g \sin \theta-\ddot{x}_{a} \cos \theta\right)$, therefore the compensation using the differential principle does not work. An hypothesis to explain these differences between simulation and experimental results is that the temperature is not uniform in the chamber which contains the two sensors and induces news disturbances that are different for each sensor (deformation of the mechanical structures supporting the external magnets and the confocal chromatic sensors).

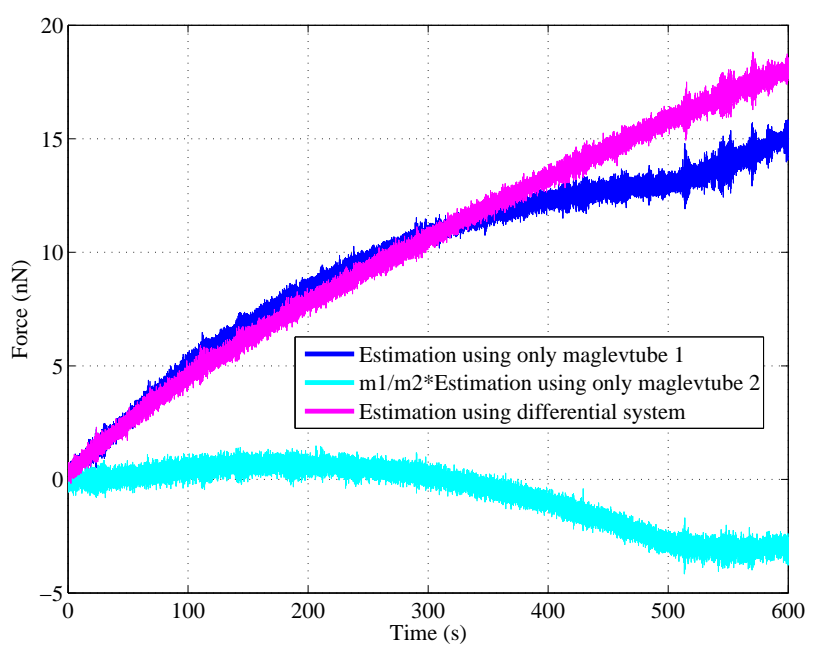

Fig. 6: Force estimation versus time for an external force $F^{x}(t)=0 \mathrm{nN}$ with environmental disturbances.

To verify this hypothesis, the temperature was monitored using two K-type wire thermocouple temperature sensors $\left(T h_{1}\right.$ and $T h_{2}$ in Fig. 3) placed close to the maglevtube deflector of each force sensor. To be sure to only measure the very low frequency impact of the temperature on the low frequency drift, the anti-vibration table was blocked to suppress the low frequency influence of the slow variation of $\theta$. Thus, the drift can not be the consequence of the terms $m_{i} g \sin \theta$ which remains constant in (6) and (7). Figure 7 shows the results of an acquisition during four days. It can be observed that the temperature evolution is slow during the week-end and more important when heater is on because of the thermal exchange between the walls of the room where the sensors are placed and the big technical hall near it. It can also be clearly seen that there is an obvious correlation between the force sensor measurements and the temperature near each deflector. Nevertheless, this correlation is different for each force sensor because the two devices used in this experiment were made at two different periods in the past and thus are based on different mechanical design and materials and do not react in the same way to temperature changes. The observed correlations mean that the hypothesis made is verified: the measurement of the two maglevtubes is sensitive to temperature changes and, with the materials used, this sensitivity is unfortunately in the same order of magnitude than the one due to the mechanical disturbance associated to $\theta$ (i.e. the term $\left.m_{1} g \sin \theta\right)$ ). Thus, it can be assumed that, in some special conditions, when the temperature is controlled and remains uniform in the chamber, the two sensors will give the same results and can be used for the compensation process.

\section{B. Compensation when temperature is uniform in the cham- ber}

The chamber in which the two sensors are enclosed is placed in an air-conditioned room in order to assure that the temperature can be uniform. After, a long period to 

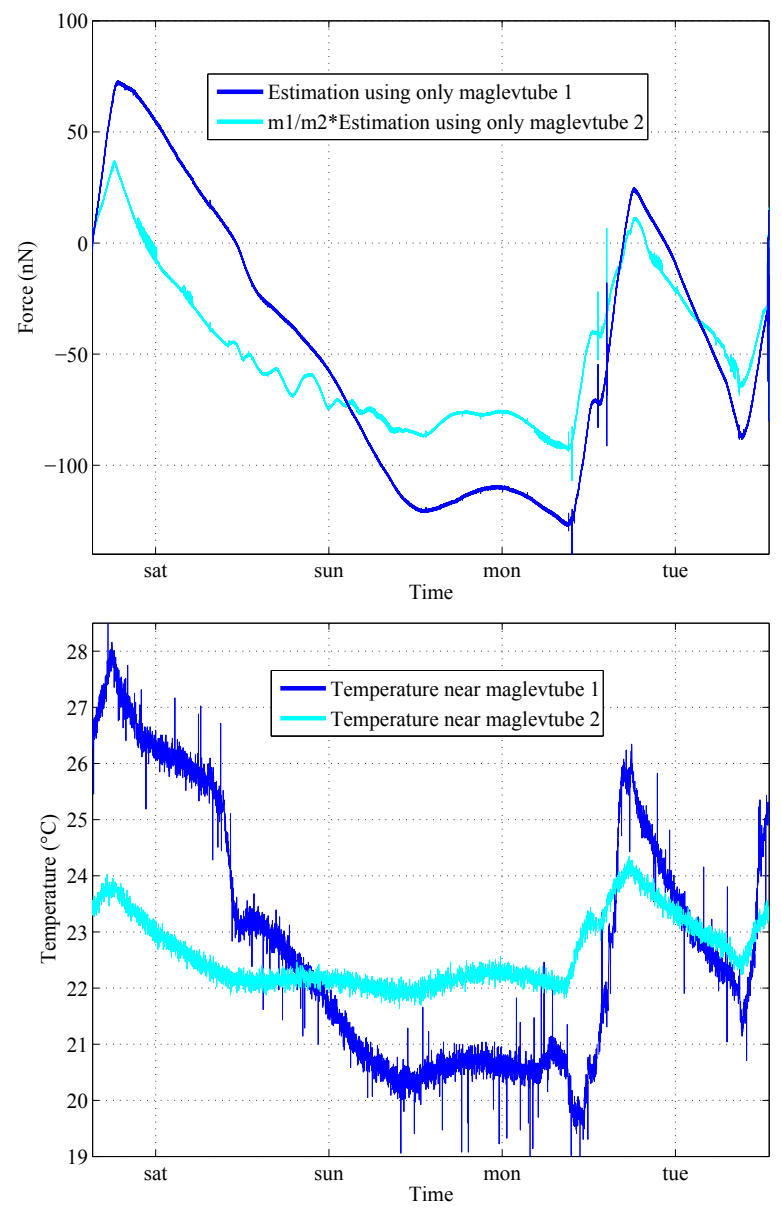

Fig. 7: Force estimation and temperature versus time for an external force $F^{x}(t)=0 \mathrm{nN}$ with environmental disturbances during 4 days.

reach the stabilization of the temperature inside the chamber, the experiments are done when the temperature is the same near the two sensors. This time, the antivibration table is not blocked any more and the same experiment as the one performed in Section $\mathrm{V}-\mathrm{A}$ is performed again to obtain Fig. 8 It shows two different experimental results obtained with the prototype placed in the air-conditioned room. They give the force estimation $\hat{F}_{k}$ when there is no external force applied on the maglevtube tip $\left(F^{x}(t)=0\right)$ and illustrates, on two different situations, the typical improvement obtained for this passive compensation principle of environmental disturbances. Figures $8 \mathrm{a}$ and $8 \mathrm{~b}$ illustrate respectively the passive compensation of the disturbances due to the slow angular variation of the anti-vibration table (effect of $\theta$ ) and due to the vibrations of the ground insufficiently filtered by the anti-vibration table (effect of $\ddot{x}_{a}(t)$ ). It can be seen, in accordance with the simulation results (Fig. 5), that despite of the movements of the anti-vibration table, the force estimated using the differential principle stays close to zero. Standard deviation in Fig. $8 \mathrm{a}$ is equal to $250 \mathrm{pN}$. Moreover, comparison between Figures $8 \mathrm{a}$ and 6 shows the importance of the temperature control to ensure a proper functioning

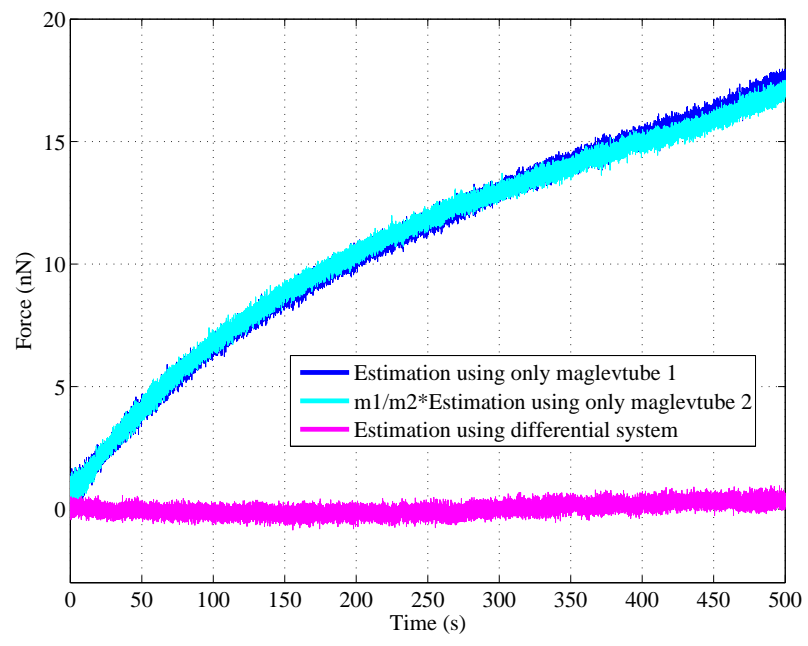

(a) $\theta$ influence

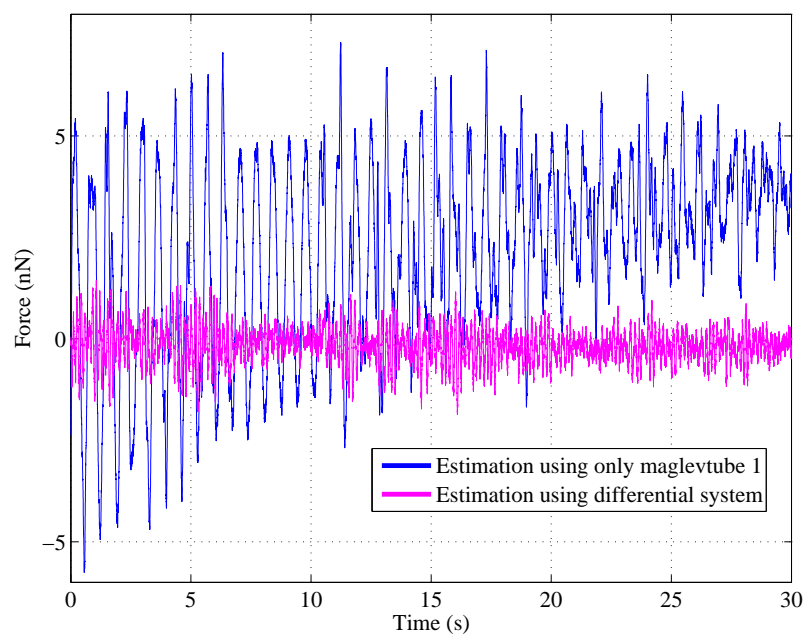

(b) $\ddot{x}_{a}(t)$ influence

Fig. 8: Force estimation (using and not using differential principle) versus time for an external force $F^{x}(t)=0 \mathrm{nN}$ with environmental disturbances.

of the compensation principle. As the mechanical structure of both sensors are different and as the materials used have a large thermal dilatation coefficient $\left(100.10^{-6} \mathrm{~K}^{-1}\right)$, the current design is not well-suited to perform differential compensation with a low sensitivity to temperature changes. Therefore the performance illustrated in Fig $8 \mathrm{a}$ remains difficult to maintain over a significant period of time and one can see that a small raising difference between the two mechanical disturbances estimations still exists for instance for time superior to 300 seconds. Figure $8 \mathrm{~b}$ illustrates the compensation of seismic disturbances with a high amplitude $\left(m_{1} \ddot{x}_{a} \cos \theta= \pm 5 \mathrm{nN}\right)$ in another experiment. In this case, the residual noise computed on a 30 seconds window has a standard deviation equal to $450 \mathrm{pN}$. This residual noise observed on $\hat{F}_{k}$ is due to two additive independent causes: (i) the residual seismic disturbances that are not totally canceled by the differential measurement principle and (ii) 


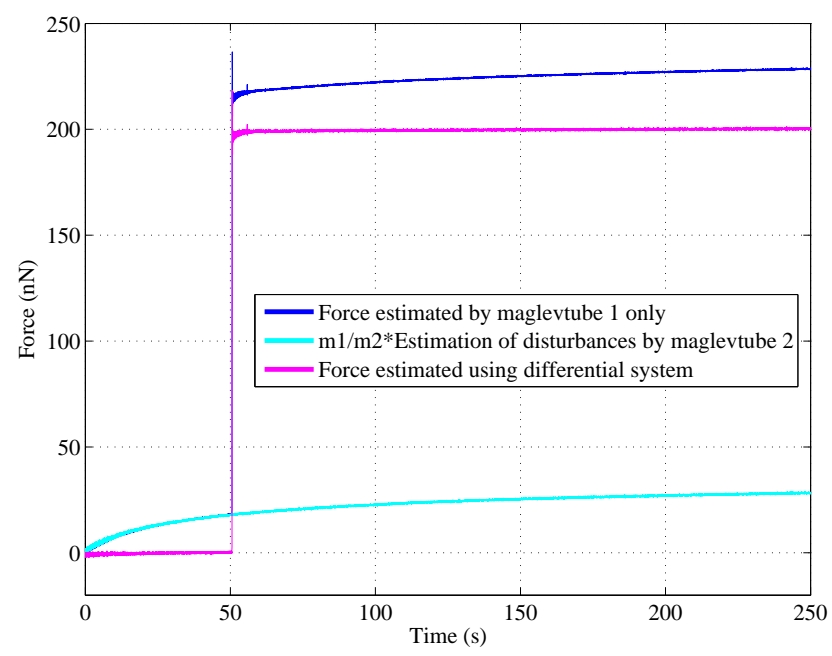

Fig. 9: Force estimation (using and not using differential principle) versus time for an external force $F^{x}(t)=200 \mathrm{nN}$ with environmental disturbances ( $\theta$ influence).

the additive residual electronic noise added by the two confocal chromatic sensors that remains after the filtering induced by the differential deconvolution process. The last figure 9 illustrates the estimation versus time of a real external force that is, this time, different from zero. This non-contact force is here a magnetic force applied on the maglevtube thanks to the coils normally used to calibrate the sensor. Such approach is useful to test the force sensor estimation with different temporal shape of external force by generating adequate current profiles in the coils. In order to better see the compensation, the anti-vibration table is touched at time $t=0$ to amplify the dynamic of the angular variation of the tabletop ( $\theta$ disturbance). At time $t=50 \mathrm{sec}$., the force step is generated (current in the coils has been adjusted to obtain an amplitude of $200 \mathrm{nN}$ ). Both force estimations using and not using the differential principle are shown. The disturbance estimation provided by the second force sensor is also provided. With the differential principle, the force estimation stays close to $200 \mathrm{nN}$, whereas it is not the case if the disturbances are not compensated.

\section{CONCLUSION}

A novel passive differential measurement principle has been presented in this article to compensate the environmental mechanical disturbances. Indeed, it has been seen in previous works that disturbances add a time-varying offset on the force estimation obtained with nanoforce sensors based on the diamagnetic levitation of a macroscopic seismic mass. Thanks to the use of two maglevtubes, this differential principle makes possible the estimation of the common low frequency and non-stationary mechanical disturbances that are applied on both transducers. The common mode rejection of these disturbances decreases the force estimation error by a significant amount on long periods of time if the environmental temperature is uniform in the chamber and should make possible to investigate, in the future, force estimations just below the nanonewton level. This approach also avoids the use of new types of sensors to estimate the disturbances effects that are added on the force (like inclinometers and accelerometers with high resolution at very low frequency). The current mechanical structure of the force sensors currently used is not optimized to minimize thermal dilatation. The choice of materials with a low sensitivity to temperature (low thermal dilatation coefficient) will be necessary for future high resolution nanoforce sensor designs using magnetic springs. Nevertheless, these first results show that this differential approach of passive compensation is promising and is more efficient for the low-end of the frequency spectrum of the mechanical disturbances (very low frequency drift due to the angular variation of the antivibration table). Improvements remain possible to have a better seismic rejection at higher frequency. A new mechanical design is currently under development to reach this goal.

\section{ACKNOWLEDGMENT}

This work has been supported by the Labex ACTION project (contract "ANR-11-LABX-01-01") and the FrenchSwiss interreg project CITHaDel.

\section{REFERENCES}

[1] P. Rougeot, S. Régnier, and N. Chaillet, "Forces analysis for micromanipulation," Proceedings 2005 IEEE international symposium on computational intelligence in robotics and automation, espoo, Finland, pp. $105-110,2005$.

[2] B. Hoogenboom, P. Frederix, D. Fotiadis, H. Hug, and A. Engel, "Potential of interferometric cantilever detection and its application for SFM/AFM in liquids," Nanotechnology, vol. 19, no. 38, p. 384019, 2008.

[3] P. Estevez, J. M. Bank, M. Porta, J. Wei, P. M. Sarro, M. Tichem, and U. Staufer, "6 DOF force and torque sensor for micro-manipulation applications," Sensors and Actuators A: Physical, vol. 186, pp. 86-93, 2012.

[4] M. Billot, X. Xu, J. Agnus, E. Piat, and P. Stempflé, "Multi-axis MEMS force sensor for measuring friction components involved in dexterous micromanipulation: Design and optimization." International Journal of Nanomanufacturing, vol. 11, no. 3/4, pp. 161-184, 2015.

[5] F. Beyeler, S. Muntwyler, and B. J. Nelson, "A six-axis MEMS forcethorque sensor with micro-newton and nano-newtonmeter resolution," Journal of Microelectromechanical Systems, vol. 18, pp. 433-441, 2009.

[6] Y. Shen, N. Xi, and W. J. li, "Contact and force control in microassembly," In IEEE 5th International Symposium on Assembly and Task Planning (ISATP), pp. 60-65, 2003.

[7] M. Boudaoud, Y. Haddab, Y. Le Gorrec, and P. Lutz, "Noise characterization in millimeter sized micromanipulation systems," Mechatronics, vol. 21, no. 6, pp. 1087-1097, 2011.

[8] J. Abadie, E. Piat, S. Oster, and M. Boukallel, "Modeling and experimentation of a passive low frequency nanoforce sensor based on diamagnetic levitation," Sensors \& Actuators: A. Physical, vol. 173, pp. 227-237, 2012.

[9] A. Cherry, E. Piat, and J. Abadie, "Analysis of a passive microforce sensor based on magnetic springs and upthrust bouoyancy," Sensors \& Actuators: A. Physical, vol. 169, pp. 27-36, 2011.

[10] J. Abadie, C. Roux, E. Piat, C. Filiatre, and C. Amiot, "Experimental measurement of human oocyte mechanical properties on a micro and nanoforce sensing platform based on magnetic springs," Sensors and Actuators B: Chemical, vol. 190, pp. 429 - 438, 2014.

[11] E. Piat, J. Abadie, and S. Oster, "Nanoforce estimation with kalman filtering applied to a force sensor based on diamagnetic levitation," in IEEE International Conference on Intelligent Robots and Systems (IROS), San Francisco, Sept. 25-30 2011, pp. 39-44.

[12] _ - "Nanoforce estimation based on kalman filtering and applied to a force sensor using diamagnetic levitation," Sensors and Actuators A: Physical, vol. 179, pp. 223-236, 2012. 